\title{
BERNSTEIN-TYPE INEQUALITIES FOR THE DERIVATIVES OF CONSTRAINED POLYNOMIALS
}

\author{
TAMÁS ERDÉLYI
}

\author{
(Communicated by R. Daniel Mauldin)
}

\begin{abstract}
Generalizing a number of earlier results, P. Borwein established a sharp Markov-type inequality on $[-1,1]$ for the derivatives of polynomials $p \in \pi_{n}$ having at most $k(0 \leq k \leq n)$ zeros in the complex unit disk. Using Lorentz representation and a Markov-type inequality for the derivative of Müntz polynomials due to $D$. Newman, we give a surprisingly short proof of Borwein's Theorem. The new result of this paper is to obtain a sharp Bernsteintype analogue of Borwein's Theorem. By the same method we prove a sharp Bernstein-type inequality for another wide family of classes of constrained polynomials.
\end{abstract}

\section{INTRODUCTION, NOTATIONS}

Markov's inequality, which plays a significant role in approximation theory and related areas, states that

$$
\max _{-1 \leq x \leq 1}\left|p^{\prime}(x)\right| \leq n^{2} \max _{-1 \leq x \leq 1}|p(x)|
$$

for every polynomial $p \in \pi_{n}$, where $\pi_{n}$ denotes the set of all real algebraic polynomials of degree at most $n$. The pointwise algebraic Bernstein-type analogue asserts that

$$
\left|p^{\prime}(y)\right| \leq \frac{n}{\sqrt{1-y^{2}}} \max _{-1 \leq x \leq 1}|p(x)| \quad(-1<y<1)
$$

for every polynomial $p \in \pi_{n}$. On every fixed subinterval $[-a, a](0<a<1)$, (2) gives a much better upper bound than (1). Let $S_{n}^{k}(z, r)$ be the family of polynomials from $\pi_{n}$ which have at most $k$ zeros in the open disk of the complex plane with center $z$ and radius $r$. A number of papers were written on Markov- and Bernstein-type inequalities for the derivatives of polynomials from $S_{n}^{k}(0,1)$ in certain special cases. When $k=0$, see [5], [6], and [9]; when $k$ is small compared with $n$, [7] and [11] give reasonable results. Finally, proving J. Szabados's conjecture, P. Borwein [1] verified the following sharp inequality:

Received by the editors January 22, 1990.

1980 Mathematics Subject Classification (1985 Revision). Primary 26C05, 26C10, 26D10.

Key words and phrases. Markov and Bernstein type inequalities, polynomials with restricted zeros. 
Theorem 1. We have

$$
\max _{0 \leq x \leq 1}\left|p^{\prime}(x)\right| \leq c n(k+1) \max _{0 \leq x \leq 1}|p(x)|
$$

for every $p \in S_{n}^{k}(1 / 2,1 / 2)$ with some absolute constant $c \leq 18$.

Using a Lorentz representation of a polynomial from $S_{n}^{0}(1 / 2,1 / 2)$ and a Markov-type inequality for the derivative of Müntz polynomials, we will present a very short proof of Theorem 1. A sharp Markov-type inequality was established in [2] for another family of classes of constrained polynomials.

Theorem 2. We have

$$
\max _{-1 \leq x \leq 1}\left|p^{\prime}(x)\right| \leq \min \left\{n^{2}, \frac{c n}{\sqrt{r}}\right\} \max _{-1 \leq x \leq 1}|p(x)|
$$

for every polynomial from $\pi_{n}$ having no zeros in the open disks with diameters $[-1,-1+2 r]$ and $[1-2 r, 1]$, respectively, where $0<r \leq 1$ and $c \leq 20$ is an absolute constant.

For $0<r \leq 1$, we define

$$
K(r)=\bigcup_{a \in[-1+r, 1-r]}\{z \in \mathbb{C}:|z-a|<r\}
$$

and denote by $W_{n}^{0}(r)$ the set of those polynomials from $\pi_{n}$ which have no zeros in $K(r)$. The main goal of this paper is to obtain sharp Bernstein-type inequalities for the derivatives of polynomials from $S_{n}^{k}(0,1)$ and $W_{n}^{0}(r)$, respectively.

\section{NeW Results}

We will prove the following Bernstein-type inequalities:

Theorem 3. We have

$$
\left|p^{\prime}(y)\right| \leq c \frac{\sqrt{n(k+1)}}{1-y^{2}} \max _{-1 \leq x \leq 1}|p(x)| \quad(-1<y<1)
$$

for every $p \in S_{n}^{k}(0,1)$, where $c$ is an absolute constant.

Theorem 4. We have

$$
\left|p^{\prime}(y)\right| \leq c \sqrt{\frac{n}{r\left(1-y^{2}\right)}} \max _{-1 \leq x \leq 1}|p(x)| \quad(-1+r<y<1-r)
$$

and

$$
\left|p^{\prime}(y)\right| \leq c \sqrt{\frac{n}{r}} \frac{1}{1-y^{2}} \max _{-1 \leq x \leq 1}|p(x)| \quad(-1<y<1)
$$

for every polynomial $p \in W_{n}^{0}(r)(0<r \leq 1)$ with certain absolute constants $c$. 


\section{THE SHARPNESS OF OUR THEOREMS}

The sharpness of Theorem 1 was proved by J. Szabados [10]. It was shown in [3] that

$$
\sup _{p \in S_{n}^{k}(0,1)} \frac{\left|p^{\prime}(0)\right|}{\max _{-1 \leq x \leq 1}|p(x)|} \geq c \sqrt{n(k+1)}
$$

with some absolute constant $c>0$.

Conjecture 1. The pointwise factor $\left(1-y^{2}\right)^{-1}$ in Theorem 3 can be replaced by $\left(1-y^{2}\right)^{-1 / 2}$.

Conjecture 2. We have

$$
\sup _{p \in W_{n}^{0}(r)} \frac{\left|p^{\prime}(0)\right|}{\max _{-1 \leq x \leq 1}|p(x)|} \geq c \sqrt{\frac{n}{r}} \quad\left(\frac{1}{n} \leq r \leq 1\right)
$$

with some absolute constant $c>0$.

Remark 1. With J. Szabados [4], we proved that

$$
\left|p^{\prime}(y)\right| \leq c \frac{\sqrt{n}(k+1)^{2}}{\sqrt{1-y^{2}}} \max _{-1 \leq x \leq 1}|p(x)| \quad(-1<y<1)
$$

for every $p \in S_{n}^{k}(0,1)$, where $c$ is an absolute constant.

\section{A NEW PROOF OF BORWEIN'S THEOREM}

In this section we give a short proof of Theorem 1. Let $\Lambda=\left\{\lambda_{j}\right\}_{j=1}^{N}$ be an increasing set of positive numbers. Denote by $\pi(\Lambda)$ the collection of $\Lambda$ polynomials of the form

$$
p(x)=a_{0}+\sum_{j=1}^{N} a_{j} x^{\lambda_{j}} \quad(0 \leq x<\infty)
$$

with real coefficients $a_{j}$. We will use the following Markov-type theorem for the derivative of $\Lambda$ polynomials.

Theorem 5 (D. Newman). For every $\Lambda$ polynomial $p$ of type (3), we have

$$
\frac{2}{3} \sum_{j=1}^{N} \lambda_{j} \leq \sup _{\pi(\Lambda)} \frac{\max _{0 \leq x \leq 1}\left|p^{\prime}(x) x\right|}{\max _{0 \leq x \leq 1}|p(x)|} \leq 11 \sum_{j=1}^{N} \lambda_{j} .
$$

The proof of Theorem 5 may be found in [8]. As a straightforward consequence of Theorem 5 , we obtain the following:

Proposition. Let $p(x)=x^{n-k} Q_{k}(x)$, where $Q_{k} \in \pi_{k}$. Then

$$
\left|p^{\prime}(1)\right| \leq 1 \ln (k+1) \max _{0 \leq x \leq 1}|p(x)| .
$$


Proof of the proposition. By Theorem 5 we have

$$
\left|p^{\prime}(1)\right| \leq 11\left(\sum_{j=n-k}^{n} j\right) \max _{0 \leq x \leq 1}|p(x)| \leq 11 n(k+1) \max _{0 \leq x \leq 1}|p(x)| .
$$

Now let $p \in S_{n}^{k}(1 / 2,1 / 2)$. By an observation of G. G. Lorentz [9], we have $p=w Q_{k}$, where $Q_{k} \in \pi_{k}$ and

$$
w(x)=\sum_{j=0}^{n-k} a_{j}(1-x)^{j} x^{n-k-j}, \text { with all } a_{j} \geq 0 .
$$

We may assume that $n-k \geq 1$; otherwise, (1) gives Theorem 1 . Using the Proposition and $a_{j} \geq 0(0 \leq j \leq n-k)$, we obtain

$$
\begin{aligned}
\left|p^{\prime}(1)\right| & =\left|\left(a_{0} x^{n-k} Q_{k}(x)\right)^{\prime}(1)+\left(a_{1}(1-x) x^{n-k-1} Q_{k}(x)\right)^{\prime}(1)\right| \\
& =\left|\left(x^{n-k-1}\left(a_{0} x Q_{k}(x)+a_{1}(1-x) Q_{k}(x)\right)\right)^{\prime}(1)\right| \\
& \leq 11 n(k+2) \max _{0 \leq x \leq 1}\left|\sum_{j=0}^{1} a_{j}(1-x)^{j} x^{n-k-j} Q_{k}(x)\right| \\
& \leq 11 n(k+2) \max _{0 \leq x \leq 1}\left|\sum_{j=0}^{n-k} a_{j}(1-x)^{j} x^{n-k-j} Q_{k}(x)\right| \\
& =11 n(k+2) \max _{0 \leq x \leq 1}|p(x)| .
\end{aligned}
$$

Now let $y \in[0,1]$ be arbitrary. To estimate $\left|p^{\prime}(y)\right|$, we may assume that $1 / 2 \leq y \leq 1$; otherwise, $P(x)=p(1-x) \in S_{n}^{k}(1 / 2,1 / 2)$ can be studied. If $p \in S_{n}^{k}(1 / 2,1 / 2)$, then $p \in S_{n}^{k}(y / 2, y / 2)$; hence, by a linear transformation, (4) yields

$$
\left|p^{\prime}(y)\right| \leq \frac{11}{y} n(k+2) \max _{0 \leq x \leq y}|p(x)| \leq 22 n(k+2) \max _{0 \leq x \leq y}|p(x)|,
$$

which finishes the proof of Borwein's Theorem.

Remark 2. Observe that $p \in S_{n}^{k}(1 / 2,1 / 2)$ does not necessarily imply $p^{\prime} \in$ $S_{n}^{k}(1 / 2,1 / 2)$; therefore, the generalization of Borwein's inequality for higher derivatives does not follow immediately from the case of the first derivative. Nevertheless, the inequality

$$
\max _{0 \leq x \leq 1}\left|p^{(m)}(x)\right| \leq c(m)(n(k+1))^{m} \max _{0 \leq x \leq 1}|p(x)|
$$

can be proved about as briefly, relying only on a Lorentz representation of $p \in S_{n}^{k}(1 / 2,1 / 2)$ and Newman's inequality.

\section{Lemmas for Theorem 3}

It is sufficient to prove Theorem 3 when $y=0$, since from this we will obtain the statement in the general case by a linear transformation. By our first lemma, we introduce an extremal polynomial $Q \in S_{n}^{k}(0,1)$. 
Lemma 1. For every $n$ and $k(0 \leq k \leq n)$ natural numbers, there exists a polynomial $Q \in S_{n}^{k}(0,1)$ such that

$$
\frac{\left|Q^{\prime}(0)\right|}{\max _{-1 \leq x \leq 1}|Q(x)|}=\sup _{p \in S_{n}^{k}(0,1)} \frac{\left|p^{\prime}(0)\right|}{\max _{-1 \leq x \leq 1}|p(x)|} .
$$

The next lemma gives some information on the zeros of the extremal polynomial $Q$ introduced by Lemma 1 .

Lemma 2. Let $Q$ be defined by Lemma 1. Then $Q$ has only real zeros, and at most $k+1$ of them are different from \pm 1 (counting multiplicities).

From Theorem 1 we will easily deduce

Lemma 3. Let $\delta=(36 n(k+1))^{-1}$. Then

$$
\max _{-\delta \leq x \leq 1}|q(x)|<2 \max _{0 \leq x \leq 1}|q(x)|
$$

for every $q \in S_{n}^{k}(1 / 2,1 / 2)$ having all its zeros in $[0, \infty)$.

From Lemma 3 we will easily obtain

Lemma 4. Let $z_{0}=i(36 n(k+1))^{-1 / 2}$ where $i$ is the imaginary unit. Then

$$
\left|p\left(z_{0}\right)\right|<\sqrt{2} \max _{-1 \leq x \leq 1}|p(x)|
$$

for every $p \in S_{n}^{k}(0,1)$ having only real zeros.

Remark 3. The inequalities of Lemmas 3 and 4 can be proved for all $p \in$ $S_{n}^{k}(1 / 2,1 / 2)$ and $p \in S_{n}^{k}(0,1)$, respectively, but under our additional assumptions their proofs are much shorter.

We will prove Theorem 3 by Cauchy's integral formula and these lemmas.

\section{LEMMAS FOR THEOREM 4}

The way to prove Theorem 4 is very similar to the previous section. We introduce an extremal polynomial by

Lemma 5. For every $n$ natural and $0<r \leq 1$ real numbers, there exists $a$ polynomial $Q \in S_{n}^{0}(0, r)(0<r \leq 1)$ such that

$$
\frac{\left|Q^{\prime}(0)\right|}{\max _{-1 \leq x \leq 1}|Q(x)|}=\sup _{p \in S_{n}^{0}(0, r)} \frac{\left|p^{\prime}(0)\right|}{\max _{-1 \leq x \leq 1}|p(x)|} .
$$

By a variational method we will obtain

Lemma 6. Let $Q$ be defined by Lemma 5. Then $Q$ has only real zeros.

From Theorem 2 we will easily prove

Lemma 7. Let $0<R \leq 1$ and $\delta=\sqrt{R} /(8 n)$. Then

$$
\max _{-\delta \leq x \leq 1}|q(x)|<2 \max _{0 \leq x \leq 1}|q(x)|
$$

for every $q \in S_{n}^{0}(0, R)$ having all its zeros in $[R, \infty)$.

Our last lemma will be a straightforward consequence of Lemma 7. 
Lemma 8. Let $0<r \leq 1$ and $z_{0}=i \sqrt{r /(8 n)}$, where $i$ is the imaginary unit. Then

$$
\left|p\left(z_{0}\right)\right|<\sqrt{2} \max _{-1 \leq x \leq 1}|p(x)|
$$

for every $p \in S_{n}^{0}(0, r)$ having only real zeros.

Remark 4. The inequalities of Lemmas 7 and 8 can be verified for all $p \in$ $S_{n}^{0}(0, R) \quad(0<R \leq 1)$ and $p \in S_{n}^{0}(r) \quad(0<r \leq 1)$, respectively, but under our additional assumptions their proofs are simpler.

We will prove Theorem 4 by Cauchy's integral formula, similarly to the proof of Theorem 3.

7. A BeRnstein-WALSh TYPe PROBLEM FOR POLYNOMials FROM $S_{n}^{k}(0,1)$

We would like some information on the magnitude of $|p(z)| \quad(z \in \mathbb{C})$ when $p \in S_{n}^{k}(0,1)$ and $\max _{-1 \leq x \leq 1}|p(x)|=1$.

Conjecture 3. Let $D$ be the ellipse of the complex plane with axes $[-a, a]$ and $[-b, b]$, where $a=1+(n(k+1))^{-1}$ and $b=i(n(k+1))^{-1 / 2}(i$ is the imaginary unit). Then there is an absolute constant $c$ such that

$$
|p(z)| \leq c \max _{-1 \leq x \leq 1}|p(x)| \quad(z \in D)
$$

for every $p \in S_{n}^{k}(0,1)(0 \leq k \leq n)$.

Conjecture 1 could be obtained immediately from Conjecture 3 and Cauchy's integral formula.

\section{Proofs of the Lemmas for Theorem 3}

The proof of Lemma 1 is a straightforward application of Hurwitz's Theorem.

Proof of Lemma 2. Assume indirectly that there are at least two zeros of $Q$ outside the open unit disk and different from \pm 1 . If $z_{1}$ is a nonreal zero of $Q$, then the polynomial

$$
Q_{\varepsilon}(x)=Q(x)-\varepsilon \frac{Q(x) x^{2}}{\left(x-z_{1}\right)\left(x-\bar{z}_{1}\right)} \in S_{n}^{k}(0,1)
$$

with a sufficiently small $\varepsilon>0$ contradicts the maximality of $Q$. If $a$ and $b$ are real zeros of $Q$ such that $|a| \geq|b|>1$, then the polynomial

$$
Q_{\varepsilon}(x)=Q(x)-\varepsilon \operatorname{sgn}(a b) \frac{Q(x) x^{2}}{(x-a)(x-b)} \in S_{n}^{k}(0,1)
$$

with a sufficiently small $\varepsilon>0$ contradicts the maximality of $Q$. Thus Lemma 2 is proved.

Lemma 3 follows immediately from Theorem 1 and the Mean Value Theorem. 
Proof of Lemma 3. Assume indirectly that there is a $-\delta \leq y<0$ such that

$$
|q(y)|=2 \max _{0 \leq x \leq 1}|q(x)|
$$

for a polynomial $q \in S_{n}^{k}(1 / 2,1 / 2)$ having all its zeros in $[0, \infty)$. Then using (5), $-\delta \leq y<0, \delta=(36 n(k+1))^{-1}$, the Mean Value Theorem, the monotonicity of $\left|q^{\prime}\right|$ on $(-\infty, 0]$, and Theorem 1 transformed linearly to the interval $[y, 1]$, we can find a $\xi \in(y, 0)$ such that

$$
\begin{aligned}
36 n(k+1) \max _{0 \leq x \leq 1}|q(x)| & \leq\left|\frac{q(y)-q(0)}{y}\right|=\left|q^{\prime}(\xi)\right| \leq\left|q^{\prime}(y)\right| \\
& \leq \frac{18}{1-y} n(k+1) \max _{y \leq x \leq 1}|q(x)| \\
& <18 n(k+1) \max _{y \leq x \leq 1}|q(x)| \\
& =36 n(k+1) \max _{0 \leq x \leq 1}|q(x)|,
\end{aligned}
$$

a contradiction.

Proof of Lemma 4. We may assume that $p \in S_{n}^{k}(0,1)$ is monic; thus, let $p(x)=\prod_{j=1}^{s}\left(x-u_{j}\right)$ with some $s \leq n$. Applying Lemma 3 to the polynomial

$$
q(x)=\prod_{j=1}^{s}\left(x-u_{j}^{2}\right) \in S_{n}^{k}\left(\frac{1}{2}, \frac{1}{2}\right),
$$

we easily deduce that

$$
\begin{aligned}
\left|q\left(-(36 n(k+1))^{-1}\right)\right| & <2 \max _{0 \leq x \leq 1}|q(x)|=2 \max _{0 \leq x \leq 1}\left|q\left(x^{2}\right)\right| \\
& =2 \max _{-1 \leq x \leq 1}|p(x) p(-x)| \leq 2\left(\max _{-1 \leq x \leq 1}|p(x)|\right)^{2} .
\end{aligned}
$$

Observe that

$$
\left|p\left(z_{0}\right)\right|^{2}=\prod_{j=1}^{s}\left(u_{j}^{2}+(36 n(k+1))^{-1}\right)=\left|q\left(-(36 n(k+1))^{-1}\right)\right|,
$$

and together with (6) this yields

$$
\left|p\left(z_{0}\right)\right|<\sqrt{2} \max _{-1 \leq x \leq 1}|p(x)| .
$$

Thus Lemma 4 is proved.

\section{Proofs of LEMMAS For TheOREM 4}

The proofs of Lemmas 5-8 are very similar to the corresponding ones from $\S 8$. The proofs of Lemmas 5 and 6 are exactly the same as those of Lemmas 1 and 2 ; therefore, we do not give any details. 
Proof of Lemma 7. We have

$$
\left|q^{\prime}(0)\right| \leq \frac{4 n}{\sqrt{R}} \max _{0 \leq x \leq 1}|q(x)|
$$

for every polynomial $q \in \pi_{n}$ having all its zeros in $[R, \infty)$. This inequality follows from the proof of Theorem 1 in [2] with a certain multiplicative constant $c$ instead of 4 . The fact that $c=4$ can be chosen was pointed out by M. v. Golitschek and G. G. Lorentz. Now assume indirectly that there exists a $-\delta \leq y<0$ such that

$$
|q(y)|=2 \max _{0 \leq x \leq 1}|q(x)|
$$

for a polynomial $q \in \pi_{n}$ having all its zeros in $[R, \infty)$. Then (9), $-\delta \leq y<0$, $\delta=\sqrt{R} /(8 n)$, the Mean Value Theorem, the monotonicity of $\left|q^{\prime}\right|$ on $(-\infty, 0]$, and $(8)$ transformed linearly to the interval $[y, 1]$ imply that there exists a $\xi \in(y, 0)$ such that

$$
\begin{aligned}
\frac{8 n}{\sqrt{R}} \max _{0 \leq x \leq 1}|q(x)| & \leq\left|\frac{q(y)-q(0)}{y}\right|=\left|q^{\prime}(\xi)\right| \leq\left|q^{\prime}(y)\right| \\
& \leq \frac{4 n}{(1-y) \sqrt{\frac{R-y}{1-y}}} \max _{y \leq x \leq 1}|q(x)| \\
& <\frac{4 n}{\sqrt{R}} \max _{y \leq x \leq 1}|q(x)|=\frac{8 n}{\sqrt{R}} \max _{0 \leq x \leq 1}|q(x)|,
\end{aligned}
$$

a contradiction.

Proof of Lemma 8. We may assume that $p \in S_{n}^{0}(0, r)$ is monic; thus, let $p(x)=$ $\prod_{j=1}^{s}\left(x-u_{j}\right)$ with some $s \leq n$. Applying Lemma 7 to the polynomial

$$
q(x)=\prod_{j=1}^{s}\left(x-u_{j}^{2}\right) \in S_{n}^{0}\left(0, r^{2}\right)
$$

we easily deduce that

$$
\begin{aligned}
\left|q\left(-\frac{\sqrt{r^{2}}}{8 n}\right)\right| & <2 \max _{0 \leq x \leq 1}|q(x)|=2 \max _{0 \leq x \leq 1}\left|q\left(x^{2}\right)\right| \\
& =2 \max _{-1 \leq x \leq 1}|p(x) p(-x)| \leq 2\left(\max _{-1 \leq x \leq 1}|p(x)|\right)^{2} .
\end{aligned}
$$

Further,

$$
\left|p\left(z_{0}\right)\right|^{2}=\prod_{j=1}^{s}\left(u_{j}^{2}+\frac{r}{8 n}\right)=\left|q\left(-\frac{r}{8 n}\right)\right|,
$$

and together with (10) this yields

$$
\left|p\left(z_{0}\right)\right|<\sqrt{2} \max _{-1 \leq x \leq 1}|p(x)|
$$

Thus Lemma 8 is proved. 


\section{Proofs of Theorems 3 AND 4}

Proof of Theorem 3. Let

$$
D=\left\{z \in \mathbb{C}:|\operatorname{Re} z| \leq \frac{1}{2},|\operatorname{Im} z| \leq \frac{1}{12}(n(k+1))^{-1 / 2}\right\} .
$$

Since $Q \in S_{n}^{k}(0,1)$ defined by Lemma 1 has only real zeros (see Lemma 2 ), from Lemma 4, by a linear transformation, we obtain

$$
|Q(z)| \leq \sqrt{2} \max _{a \leq x \leq b}|Q(x)| \leq \sqrt{2} \max _{-1 \leq x \leq 1}|Q(x)| \quad(z \in D),
$$

where, with the notation $y=\operatorname{Re} z,[a, b]=[-1,1+2 y]$ if $-\frac{1}{2} \leq y \leq 0$, and $[a, b]=[2 y-1,1]$ if $0 \leq y \leq \frac{1}{2}$. Now let $S$ be the circle with center 0 and radius $(n(k+1))^{-1 / 2} / 12$. By Cauchy's integral formula, (12), and (13), we obtain

$$
\begin{aligned}
\left|Q^{\prime}(0)\right| & =\frac{1}{2}\left|\int_{S} \frac{Q(\xi)}{\xi^{2}} d \xi\right| \leq \frac{1}{2} \int_{S}\left|\frac{Q(\xi)}{\xi^{2}}\right||d \xi| \\
& \leq \pi \frac{1}{12}(n(k+1))^{-1 / 2}\left(12(n(k+1))^{1 / 2}\right)^{2} \sqrt{2} \max _{-1 \leq x \leq 1}|Q(x)| \\
& \leq c \sqrt{n(k+1)} \max _{-1 \leq x \leq 1}|Q(x)| .
\end{aligned}
$$

From this and the maximality of $Q$, we deduce

$$
\left|p^{\prime}(0)\right| \leq c \sqrt{n(k+1)} \max _{-1 \leq x \leq 1}|p(x)| \quad\left(p \in S_{n}^{k}(0,1)\right),
$$

and from here we obtain the desired result in the general case $-1<y<1$ by a linear transformation. Thus Theorem 3 is proved.

Proof of Theorem 4 . We proceed in a very similar way. Let

$$
D=\left\{z \in \mathbb{C}:|\operatorname{Re} z| \leq \frac{r}{2},|\operatorname{Im} z| \leq \sqrt{\frac{r}{32 n}}\right\} .
$$

Since $Q \in S_{n}^{0}(0, r)$ defined by Lemma 5 has only real zeros (see Lemma 6), from Lemma 8 , by a linear transformation, we deduce

$$
|Q(z)| \leq \sqrt{2} \max _{-1 \leq x \leq 1}|Q(x)| \quad(z \in D) .
$$

Let $S$ be the circle with center 0 and radius $\sqrt{r /(32 n)}$. Since $r \geq 1 / n$, we have $\sqrt{r /(32 n)} \leq r / 2$; hence, by Cauchy's integral formula, (14), and (15), we get

$$
\begin{aligned}
\left|Q^{\prime}(0)\right| & =\frac{1}{2}\left|\int_{s} \frac{Q(\xi)}{\xi^{2}} d \xi\right| \leq \frac{1}{2} \int_{S}\left|\frac{Q(\xi)}{\xi^{2}}\right||d \xi| \\
& \leq \pi \sqrt{\frac{r}{32 n}} \frac{32 n}{r} \sqrt{2} \max _{-1 \leq x \leq 1}|Q(x)| \leq c \sqrt{\frac{n}{r}} \max _{-1 \leq x \leq 1}|Q(x)| .
\end{aligned}
$$


Therefore the maximality of $Q$ yields

$$
\left|p^{\prime}(0)\right| \leq c \sqrt{\frac{n}{r}} \max _{-1 \leq x \leq 1}|p(x)| \quad\left(p \in S_{n}^{0}(0, r)\right) .
$$

Now observe that $p \in W_{n}^{0}(r)$ implies $p \in S_{n}^{0}(y, r)$ if $y \in[-r, r]$, and $p \in$ $S_{n}^{0}(y, 1-y)$ if $r \leq|y|<1$. Applying (17) transformed linearly to the interval $[2 y-1,1]$ when $0 \leq y \leq 1$ and $[-1,2 y+1]$ when $-1 \leq y<0$, we obtain (i) and (ii) immediately. Thus Theorem 4 is proved.

\section{REFERENCES}

1. P. Borwein, Markov's inequality for polynomials with real zeros, Proc. Amer. Math. Soc. 93 (1985), 43-47.

2. T. Erdélyi, Markov-type estimates for certain classes of constrained polynomials, Constr. Approx. 5 (1989), 347-356.

3. __ Pointwise estimates for derivatives of polynomials with restricted zeros, Colloq. Math. Soc. J. Bolyai 49; Alfred Haar Memorial Conference (Budapest, 1985), North-Holland, Amsterdam and Budapest, 1987, pp. 329-343.

4. T. Erdélyi and J. Szabados, Bernstein-type inequalities for a class of polynomials, Acta. Math. Hung. 53 (1989), 237-251.

5. P. Erdös, On extremal properties of the derivatives of polynomials, Ann. of Math. 41 (1940), 310-313.

6. G. G. Lorentz, Degree of approximation by polynomials with positive coefficients, Math. Ann. 151 (1963), 239-251.

7. A. Máté, Inequalities for derivatives of polynomials with restricted zeros, Proc. Amer. Math. Soc. 88 (1981), 221-225.

8. D. J. Newman, Derivative bounds for Müntz polynomials, J. Approx. Theory 18 (1976), 360-362.

9. J. T. Scheick, Inequalities for derivatives of polynomials of special type, J. Approx. Theory 6 (1972), 354-358.

10. J. Szabados, Bernstein and Markov type estimates for the derivative of a polynomial with real zeros, Functional Analysis and Approximation, Birkhäuser Verlag, Basel, 1981, pp. 177-188.

11. J. Szabados and A. K. Varma, Inequalities for derivatives of polynomials having real zeros, Approximation Theory III (E. W. Cheney, ed.), Academic Press, New York, 1980, pp. 881-888.

Department of Mathematics, The Ohio State University, Columbus, Ohio 43210 